\title{
Morphological basis of radiological band shadows on chest radiographs
}

\author{
AMANDA HERBERT, GERARD SLAVIN, LOUIS KREEL, BRENDA SANDIN, \\ AND CHRISTINE BATEMAN
}

From the Departments of Histopathology and Radiology, Northwick Park Hospital and Clinical Research
Centre, Harrow, Middx.

SYNOPSIS Lungs of unselected cadavers were fixed at necropsy using a formalin vapour technique. $\subseteq$ 'Band shadows' were identified in the excised lungs and these were correlated with in vivo radiographs and with the morphological changes in the lung. Persistent shadows were produced by $\overrightarrow{0}$ pulmonary infarction, subsegmental atelectasis, and septal fibrosis singly and in combination. 은 Potentially transient shadows were seen in association with atelectasis and pulmonary oedema.

Band shadows are linear intrapulmonary shadows seen on chest radiographs that may be up to several millimetres in width and $30-70 \mathrm{~mm}$ in length. They may be transient, such as the horizontal linear shadows often seen above the diaphragm in postoperative and postpartum chest radiographs which are not seen in subsequent films, or they may persist on sequential chest radiographs over a long period.

It is possible to infer the origin of certain band shadows during life, for example by the demonstration of an obstructive bronchial lesion proximal to the band shadow caused by an atelectatic segment of lung, or by observing the evolution of a solid infarct shadow into a thin band on sequential chest radiographs in a patient with arteriographic evidence of pulmonary embolism. However, much of the radiological evidence for the origin of band shadows has been circumstantial and not based on anatomical study.

There are, moreover, difficulties in the retrospective diagnosis of band shadows by the examination of lungs at necropsy: the pathologist is usually not informed of changes in the chest radiographs, and examination of uninflated lungs may be inadequate to demonstrate foci of atelectasis or small scars. Examination of lungs distended with liquid formalin may demonstrate macroscopic lesions, but small lesions can be overlooked or not examined histologically unless the pathologist is aware of their radiological significance. Transient band shadows occur as incidental non-fatal episodes and present a further problem: only close co-operation of radio-

Received for publication 26 November 1975 logist and pathologist, with postmortem radio- $\vec{\theta}$ graphic examination of lungs, ensures that such? transient lesions are recognized and examined.

A direct comparison of radiological and pathological findings was carried out by Fleischner et alo (1941). Their heroic method demanded the sus-气 pension of the body by a rope and pulley system. $\stackrel{\unrhd}{\varrho}$ Postmortem chest radiographs of the suspended $\overrightarrow{\vec{A}}$ cadaver were then taken and the lungs were examined 3 pathologically after instillation of formalin. They? showed that infarct scars could produce linearo shadows and they also verified the concept of 'plate atelectasis' underlying transient horizontal linearo shadows above the diaphragm. The development of a method of routine radiography of inflated, excised lungs permits correlations more easily (Wright $e t$ al, 1974). In this paper we report the pathological features of lungs bearing radiological band shadows 5 investigated by this technique.

\section{Material and methods}

The lungs of unselected cadavers were examined at necropsy. Lungs were removed complete with maine bronchi and were inflated by formalin vapour using intermittent positive pressure ventilation (Wrigh을 et al, 1974). Anteroposterior and lateral radiographs were taken of the fixed lungs. The lungs were then ${ }^{+}$ sliced to a thickness of $10-15 \mathrm{~mm}$ and further radiographs were taken of the slices. Histological blocks $\frac{\text { Dे }}{\mathrm{D}}$ were selected by the pathologist in association with? the radiologist after inspection of the lungs and both in vivo and necropsy radiographs. The exact loca? tions of the blocks were marked on the lung slices 
radiographs. Multiple sections were cut through the blocks as required and direct correlations were made between histological sections, postmortem radicgraphs, and chest radiographs taken duringlife. Band shows identified only on the necropsy radiographs were also directly correlated with the histology. In this way some potentially reversible lesions present at the time of death were examined.

\section{Results}

The pathological lesions underlying 10 band shadows in nine patients are described in detail below and summarized in the table. In six cases persistent band shadows were followed from in vivo films to necropsy radiographs. Four were caused by long-standing lesions and two by acute, potentially reversible lesions. In four cases band shadows present on necropsy radiographs were not identified on previous in-vivo chest radiographs: two were caused by acute potentially reversible lesions and two by longstanding lesions.

GROUP 1 BAND SHADOWS ASSOCIATED WITH PULMONARY INFARCTION

Case 1

E.P., a 79-year-old woman, a diabetic of 30 years' duration, was admitted for amputation of a gangrenous toe. On admission the chest radiograph showed an oblique band shadow $70 \times 20 \mathrm{~mm}$ above the right hemidiaphragm (fig 1) which persisted four weeks later when a further left basal band shadow appeared after a presumed pulmonary embolism. She was treated with anticoagulants but collapsed and died 16 days later.

At necropsy there was a recent myocardial infarct and thrombosis of the left circumflex coronary artery. There was thrombosis of multiple veins in the muscles of the right calf. The combined weight of the unfixed lungs was $995 \mathrm{~g}$.

Radiographs of the vapour-fixed lungs showed an oblique band shadow in the posterior lower lobe of the right lung corresponding to the persistent shadow in the in vivo chest radiograph. There were smaller band shadows lying anteriorly in the lingula, and below this there was a wedge of consolidated lung.

The lesions underlying the right basal band shadow, seen on the whole inflated lung as contracted subpleural scarring (fig 2), consisted of two adjoining brown scars. Posteriorly, a rectangular scar extended from the lateral pleural surface, which was smooth and indented, and anteriorly there was scarring at the costodiaphragmatic angle with deep indrawing of the pleura (fig 3). The histology of both lesions showed fibrotic scars associated with throm-

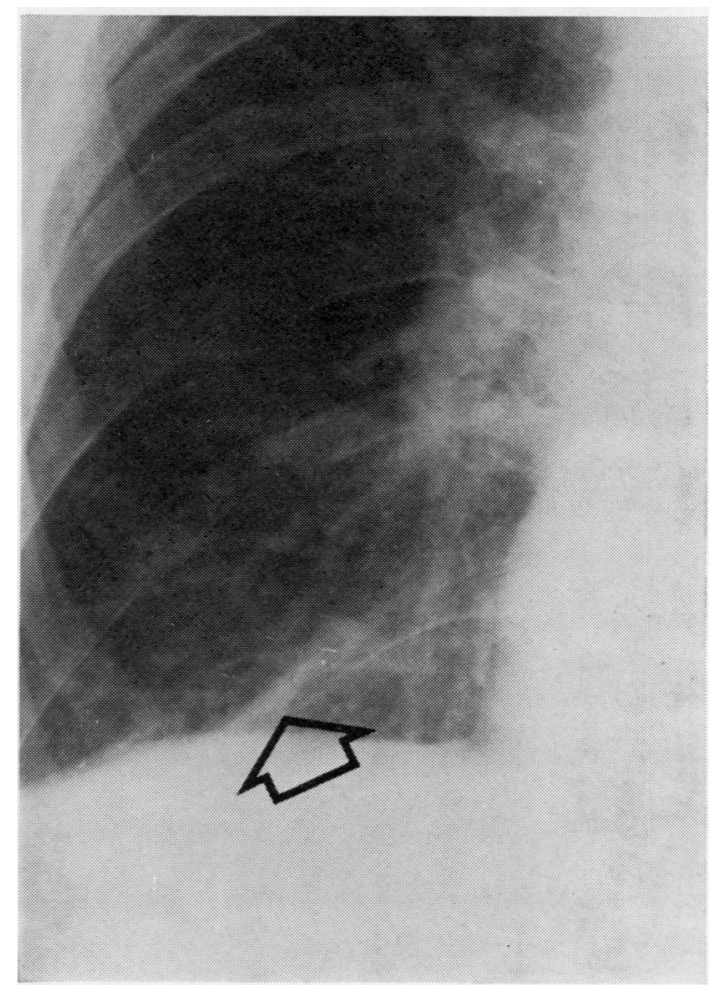

Fig 1 Case 1. Oblique linear band shadow is seen at right base in in vivo chest radiograph.

\begin{tabular}{llll}
\hline Group & Band Shadows & Case No. & Aetiological Factor \\
\hline I & Associated with pulmonary infarction & 1 & Organized infarction; atelectasis \\
& & 2 & Recent infarction; bronchiolitis; atelectasis \\
& & 3 & Organized infarction \\
II & Associated with atelectasis & 4 & Organized infarction; atelectasis \\
& & 5 & (a) Atelectasis \\
& & (b) Bronchiolitis; atelectasis \\
III & Associated with interstitial septal oedema and/or fibrosis & 6 & Bronchiolitis; atelectasis \\
IV & Associated with multiple factors & 7 & Septal oedema (D-line) \\
& & 9 & Septal fibrosis \\
\end{tabular}

Table Aetiological factors in band shadows 


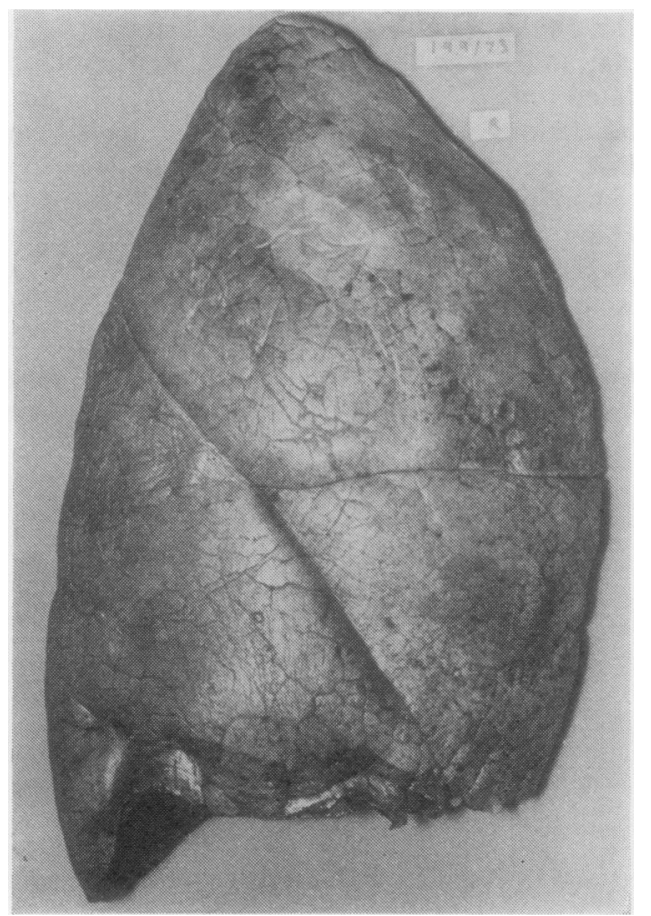

Fig 2 Case 1. Inflated right lung showing scarring with indrawing of pleura in the lower lobe. bosed and recanalized pulmonary arteries (fig 4). The alveolar architecture was lost and the residual elastic fibres showed the 'tangled hair' pattern typical of old infarcts (Castleman, 1940). Adjacent to the anterior scar a wedge of simple atelectasis exaggerated the deep indrawing of the pleura at the upper margin of the scar (fig 3).

On slicing the left upper lobe, linear scars were not seen. There was a solid fibrotic lesion in the lingula with an irregular though not indrawn pleural surface. Microscopy of this area showed an organized pneumonia. The alveolar architecture was maintained with intra-alveolar fibrosis. No evidence of infarction or atelectasis was seen. Linear scars were not seen on the radiographs of the sliced lungs and the linear shadowing seen in life was not explained. In addition to the fibrotic scarring both lungs showed severe intra-alveolar oedema and there were fresh emboli in segmental branches of the pulmonary arteries.

\section{Case 2}

M.J., a 75-year-old woman, was admitted with acute myocardial infarction. Five days later she developed an arrhythmia and died.

Necropsy confirmed an anterior myocardial infarct with thrombosis of the anterior descending coronary artery. The combined weight of the unfixed lungs was $750 \mathrm{~g}$.

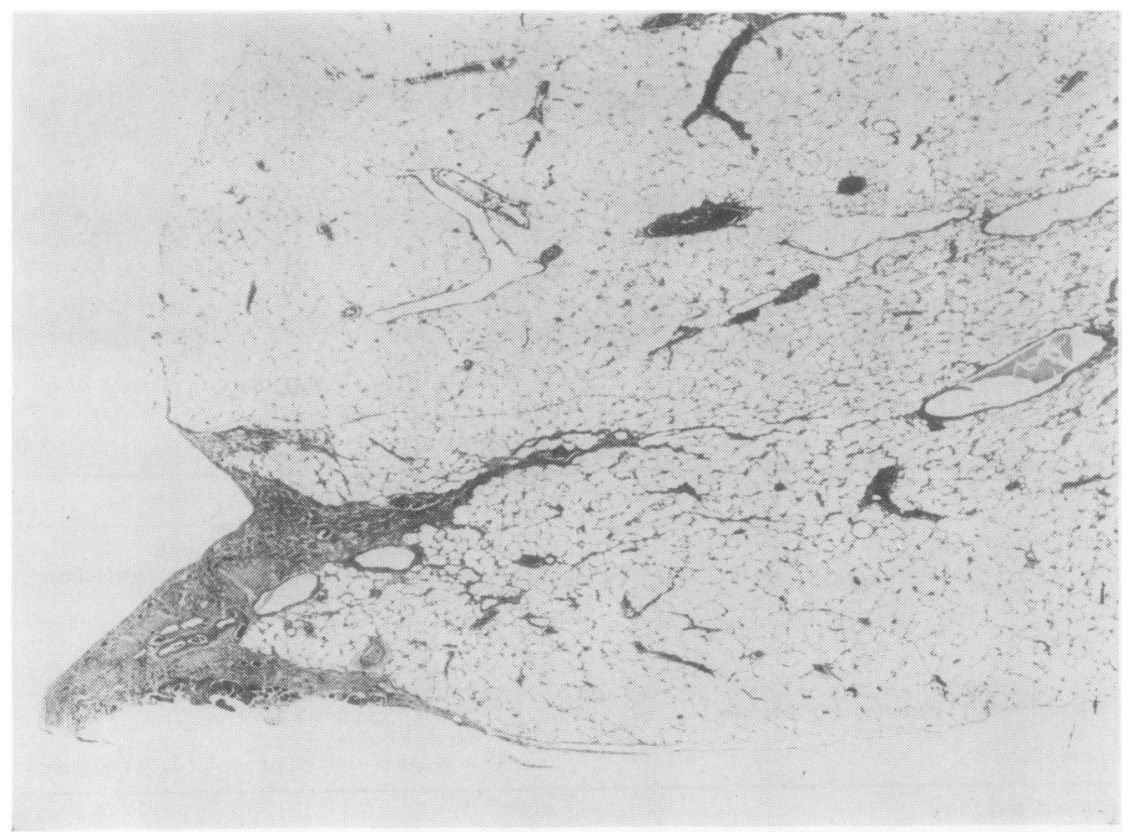

Fig 3 Case 1. Infarct scar at the costodiaphragmatic angle with linear atelectasis which accentuates pleural retraction. Haematoxylin and $\operatorname{eosin} \times 3$. 


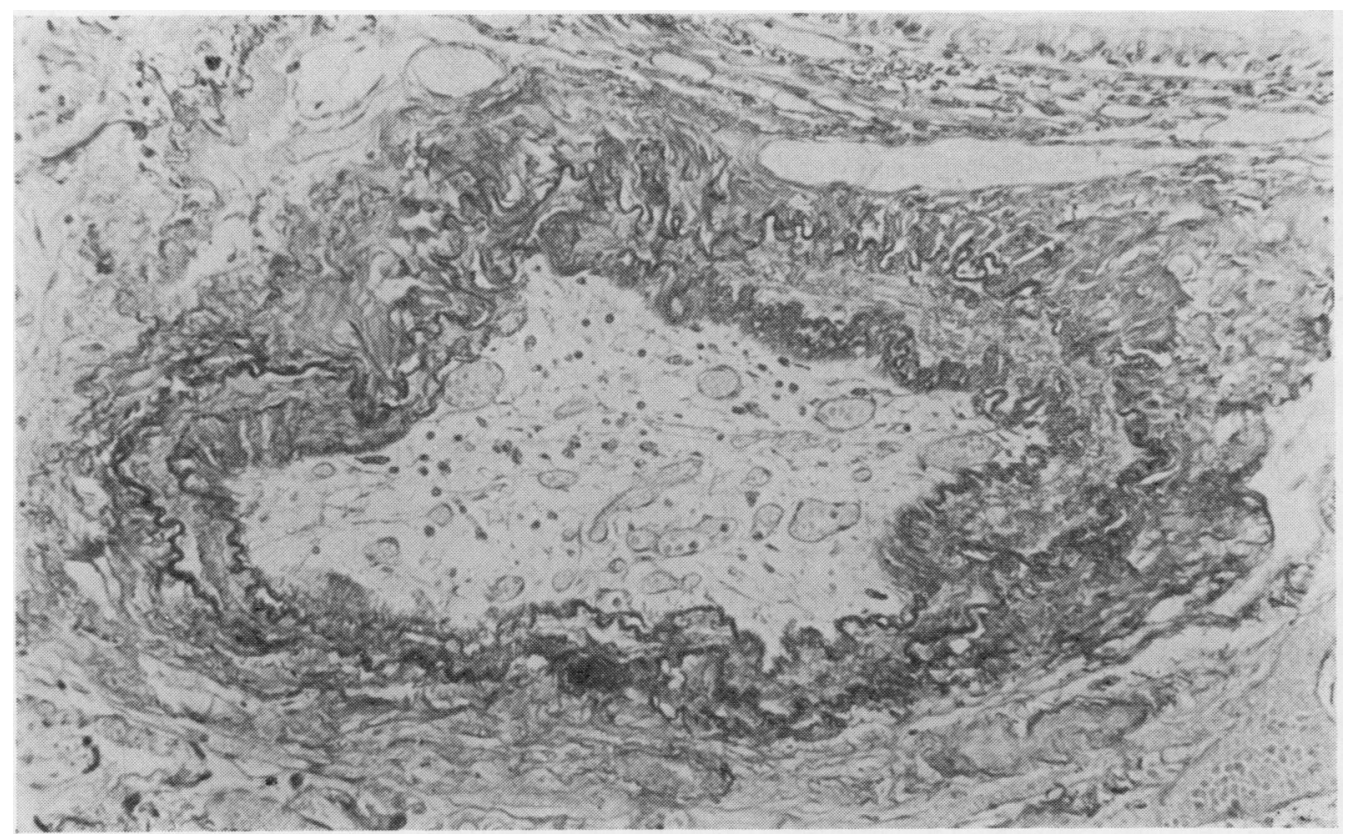

Fig 4

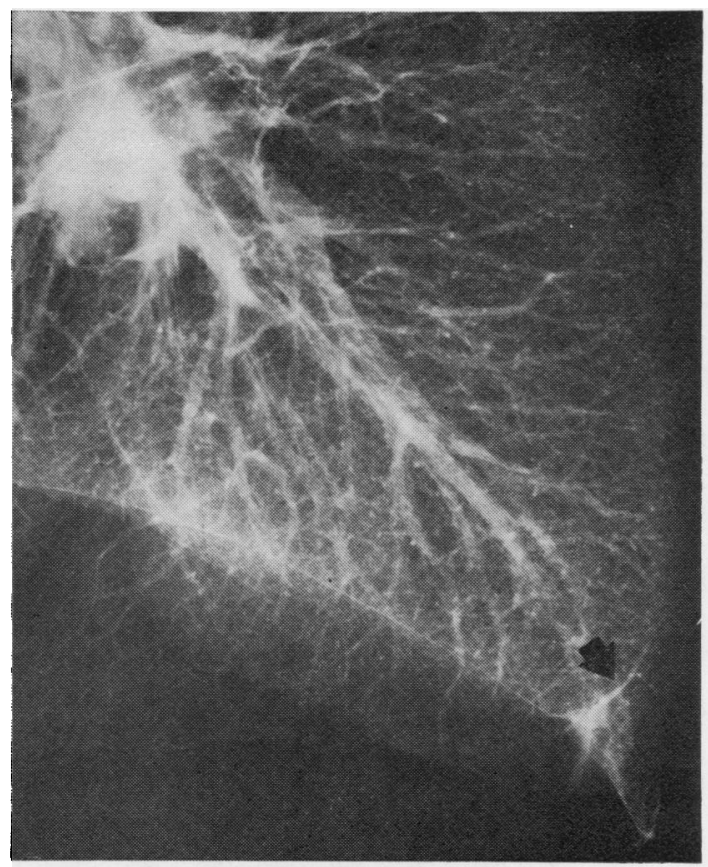

Fig 4 Case 1. Thrombosed artery showing recanalization Elastic van Gieson × 195.

Fig 5 Case 2. Radiograph of lung slice shows a band shadow (arrow) in the posterior basal segment of lung.

Fig 5 
Radiographs of the inflated lung showed a small band shadow in the posterior basal segment measuring $32 \times 2 \mathrm{~mm}$. The band shadow ran vertically and was associated with pleural retraction (fig 5). The appearances were consistent with a healed basal pulmonary infarct. This small band shadow was not seen on review of in vivo chest radiographs.

On slicing the lung there was a small contracted haemorrhagic lesion at the costodiaphragmatic angle of the right posterior base of the lower lobe. Sections showed an acute pulmonary infarct undergoing organization. There was also acute purulent bronchiolitis. The infarction involved the costodiaphragmatic angle and also a thin subpleural strip of the lateral costal cortex which showed marked atelectasis. It was this subpleural extension of the infarct which produced the linear shadow seen on the radiograph. The degree of parenchymal collapse seen in this lesion was unusual for an acute pulmonary infarct and may have been due to the associated bronchiolitis.

\section{Case 3}

H.C., a 92-year-old man, with an untreated carcinoma of bladder, developed terminal acute pyelonephritis and bronchopneumonia.

At necropsy the combined weight of the unfixed lungs was $1055 \mathrm{~g}$.

Radiographs of the inflated lungs showed a short line shadow $21 \times 2 \mathrm{~mm}$, with ill-defined margins and retraction of the adjacent pleural surface, on the anterior aspect of the middle lobe. Bilateral basal pulmonary oedema and scattered bronchopneumonic shadowing were also present, and several $1 \mathrm{~cm}$ metastases were seen in the left lower lobe.

On slicing the lungs there was basal oedema and bronchopneumonia with scattered fresh emboli in segmental pulmonary arteries. There was a small fibrotic scar in the anterior middle lobe corresponding to the band shadow. Histological sections showed scarring and fibrosis with the fragmented elastica of an old infarct, and surrounding compensatory emphysema.

\section{Case 4}

H.A., a 68-year-old man, died with widespread metastases from carcinoma of the bladder.

At necropsy the lungs weighed $770 \mathrm{~g}$.

Radiographs of the excised inflated lungs showed multiple interstitial oedema lines, but there was also a vertical-oblique band shadow lying posteromedially at the base of the right lower lobe, measuring $83 \times 3 \mathrm{~mm}$. There was retraction of the pleural surface of the lung. Multiple subpleural metastases were also visible. The band shadow was not visible on the in vivo chest radiographs.
On slicing the lungs there were multiple sub- $\frac{\stackrel{0}{*}}{2}$ pleural and parenchymal metastases. The lesion $\frac{\text { o }}{2}$ producing the band shadow was a contracted scar $\overrightarrow{\vec{F}}$ extending between opposing pleural surfaces poster- $\frac{\mathcal{O}}{O}$ iorly at the base of the right lower lobe with indraw- $\frac{\bar{C}}{0}$ ing of the pleura. Histological sections showed a $\frac{\bar{\sigma}}{\bar{c}}$ small organized infarct with recanalized arteries $\stackrel{\widetilde{\sigma}}{\widetilde{\sigma}}$ lying beneath the indrawn pleura. Extending from $\propto$ the scar to the opposite pleura was a band of क simple atelectasis.

GROUP 2 BAND SHADOWS ASSOCIATED

WITH ATELECTASIS ALONE

Case 5

S.G., an 82-year-old man, whose chest radiographs had previously been normal, was admitted for a. $v$ transurethral resection for prostatic carcinoma. $\stackrel{9}{-}$ Chest radiography showed a short thick band ${ }^{\circ}$ shadow measuring $28 \times 2 \mathrm{~mm}$ across the left은 costophrenic angle. He was readmitted three months $\vec{C}$ later with bronchopneumonia and died five days later.

At necropsy there was cerebral atrophy, old $\vec{\varphi}$ cerebral infarction, and prostatic adenocarcinoma. The combined weight of the unfixed lungs was $880 \mathrm{~g}$.

Radiographs of the inflated lungs showed a short band shadow $22 \times 1 \mathrm{~mm}$ in the anterior part of the inferior segment of the lingula, which also had $\stackrel{\complement}{\Phi}$ a vertical component forming an inverted T (fig 6). $\overrightarrow{\vec{\rho}}$ On the lateral view there was marked pleural 3 retraction which indented the pleura for $32 \mathrm{~mm}$ and was $21 \mathrm{~mm}$ wide at the pleural surface. There waso also a short oblique band shadow at the right base $(28 \times 1.5 \mathrm{~mm})$.

On slicing both lungs focal emphysema, acute $\frac{0}{3}$ bronchiolitis, and pulmonary oedema were found. The left lung showed atelectasis in the inferior segment of the lingula over which the pleural surface was smooth and deeply indented although the distal tip of the lingula was expanded normally $\frac{D}{O}$ (fig 7). Vertical blocks were taken of the inferior? lingula to include the entire lesion as well as proxi- N mal and distal lung (fig 8). The lung parenchyma showed simple atelectasis with moderate elastic thickening of the alveolar walls (fig 9). The bronchic and pulmonary arteries proximal and distal to the lesion were quite normal. There was no fresh oro recanalized thrombus: no cause for the atelectasis was found.

The right lung showed a firm, linear lesion at the lateral costodiaphragmatic angle of the lower lobe. $\frac{O}{\mathbb{D}}$ Microscopy showed a zone of atelectasis with focal? pleural and septal fibrosis. A proximal bronchiole $\mathbb{Q}$ was plugged with pus, suggesting that the atelectasis was of recent origin due to bronchiolar obstruction. 


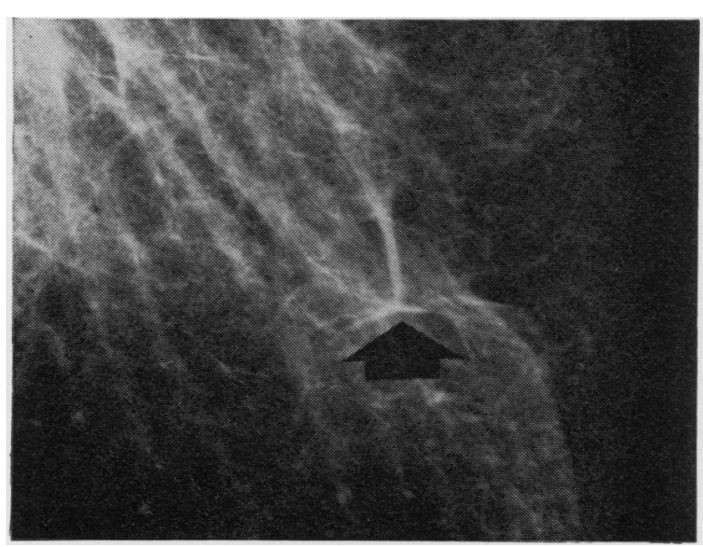

Fig 6 Case 5. Radiograph of the inflated, unsliced lung with a band shadow forming an inverted $\mathrm{T}$ in the lingula.

\section{Case 6}

J.D., a 59-year-old man, had a carcinoma of the rectum treated by abdominoperineal resection. Two years later he developed metastases in the liver, brain and lung. He deteriorated rapidly and died within two months. Chest radiography showed a short horizontal band shadow in the left midzone $(31 \times 2 \mathrm{~mm}$ ) towards the periphery (fig 10).

At necropsy widespread metastatic adenocarcinoma was confirmed. The combined weight of the unfixed lungs was $1315 \mathrm{~g}$.

Radiographs of inflated lungs showed bilateral basal pulmonary oedema which rose above the lesser fissure on the right side. Multiple ' $B$ ' and ' $D$ ' lines of interstitial pulmonary oedema were also noted as well as multiple nodular metastases. A short horizontal band shadow was present at the periphery of the left mid-zone on the posterior slice of the left lung.

When sliced, the lungs showed extensive cavitating metastases. In the posterolateral cortex of the left lower lobe there was a firm linear lesion with a deeply indrawn pleural margin (fig 11). Histology showed a wedge of acute atelectasis associated with bronchiolitis and bronchopneumonia. There was no fibrosis or scarring and no evidence of infarction or pulmonary embolism.

GROUP 3 BAND SHADOWS ASSOCIATED

WITH OEDEMA OR FIBROSIS OF THE

INTRALOBAR CONNECTIVE TISSUE

Numerous cases were seen in which interstitial oedema was sufficiently severe to produce broad band shadows not conforming to Kerley's A, B, or $\mathrm{C}$ lines. These are illustrated by one case.

\section{Case 7}

E.H., a 25-year-old woman, had had chronic myeloid leukaemia for two years. One year before death she had a splenectomy and in the last month of life developed a 'blast' crisis with terminal pseudomonas septicaemia and shock. Numerous 'band shadows' were seen in the right midzone and base (fig 12).

At necropsy there was very little remaining haemopoietic tissue. Pseudomonas species was cultured from the brain and cerebrospinal fluid. The combined weight of the unfixed lungs was $1500 \mathrm{~g}$.

Radiographs of the formalin-inflated lungs clearly

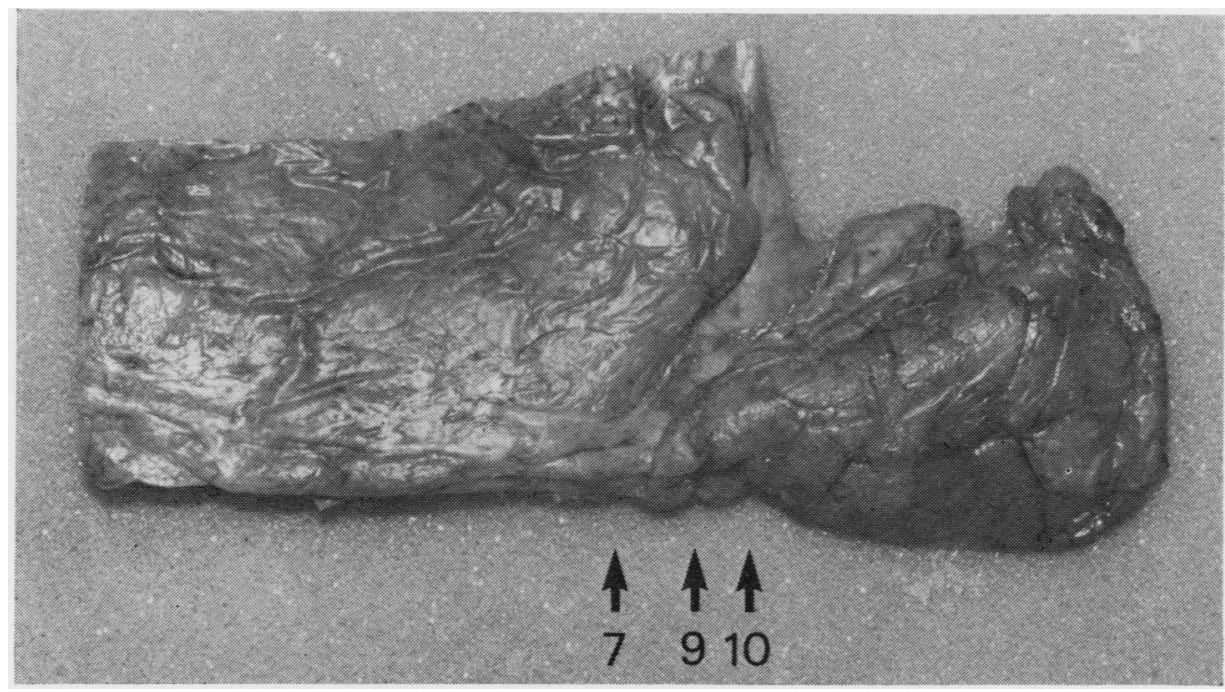

Fig 7 Case 5. Inferior segment of lingula with a depressed scar and marked indrawing of the pleura. 


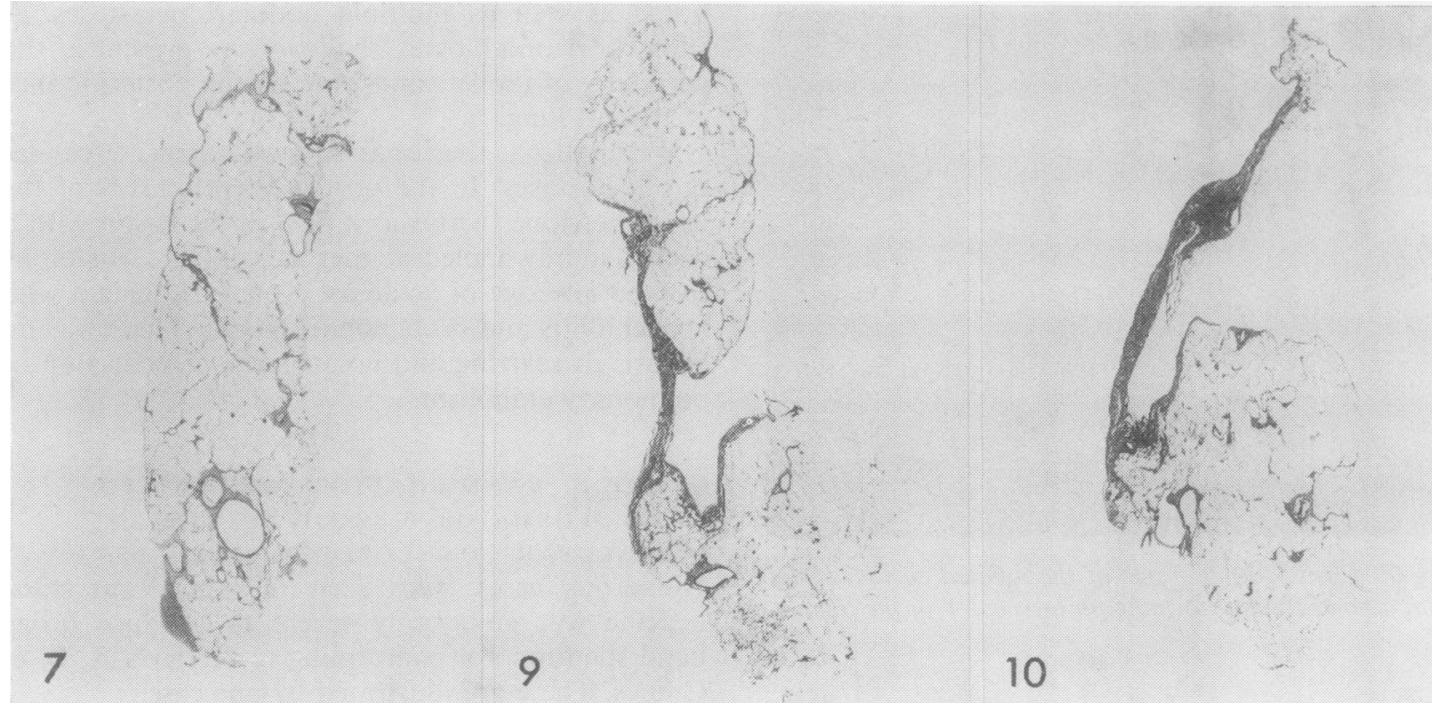

Fig 8 Case 5. Sections taken at the levels marked in fig 7, showing atelectasis: the bronchovascular bundle supplying the affected lung is normal. EVG $\times 2$.

demonstrated numerous Kerley $\mathbf{B}$ lines, but in addition the thick band-like shadows were also identified (fig 13).

Matching histological sections showed that these latter lines were due to broad markedly oedematous septa running from the pleural surface and branching to form an irregular network between veins and bronchovascular bundles (fig 14). Some of the septa contained distended lymphatics. There was focal, but $\frac{\mathrm{D}}{\circ}$ extensive, intra-alveolar oedema.

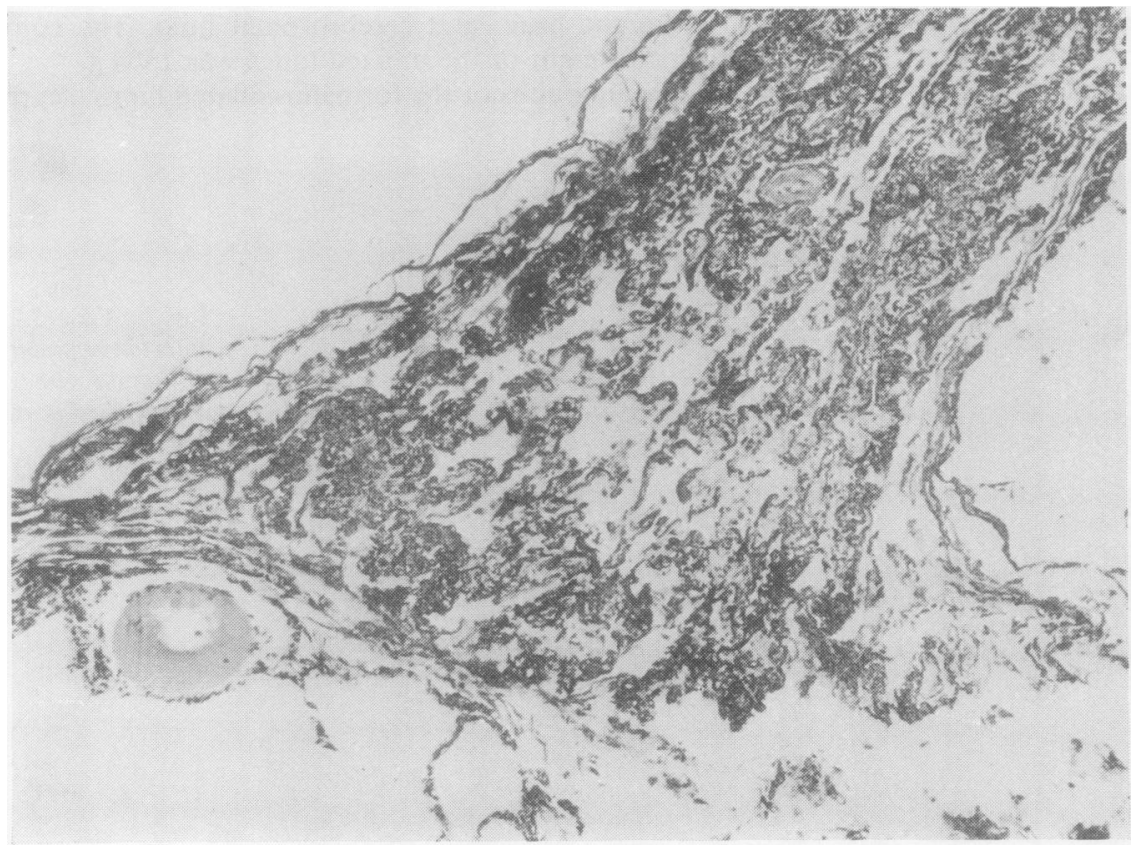

Fig 9 Case 5. Detail of section 9 in fig 8 , showing longstanding atelectasis with maintained alveolar architecture and no fibrosis. EVG $\times 30$. 


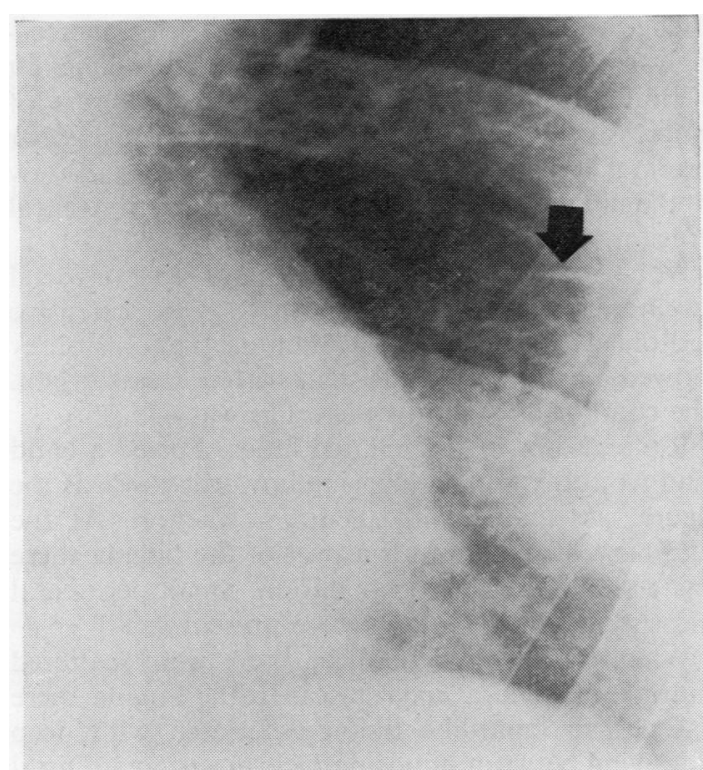

Fig 10. Case 6. Horizontal band shadow in the left mid-zone.

Fibrosis of connective tissue septa was seen in case 8.

\section{Case 8}

F.B., a 65-year-old man, was treated with intermittent chemotherapy for nine months before he died from multiple myeloma. One month before death he developed increasing back pain, necessita- ting radiotherapy. He developed bilateral basal bronchopneumonia and died in acute renal failure. The chest radiographs during his terminal illness showed a short, thick horizontal band shadow across the left costophrenic angle which appeared as a long, thin band, $85 \times 2 \mathrm{~mm}$, in the posterior segment of the left lower lobe on the lateral view.

Necropsy confirmed multiple myeloma with 'myeloma kidney'. There was a dense localized pleural thickening of the diaphragmatic pleura which bound the lung to the diaphragm.

Frontal radiographs of the inflated lungs showed a short horizontal band at the periphery of the left base merging with markedly thickened basal pleura. In the lateral film it appeared as a long linear shadow just above markedly indented pleura below which there was a dense area of thickening.

The lung slices showed a fibrotic septum running obliquely from grossly thickened pleura for $25 \mathrm{~mm}$ into the lung. Histology showed that the fibrotic septum and pleura both consisted of mature collagen with no cellular infiltrate (fig 15). There was no infarction or atelectasis. The adjacent lung parenchyma appeared normal.

\section{GROUP 4 BAND SHADOW PRODUCED BY A COMPLEX COMPOSITE LESION \\ Case 9}

L.D., a 56-year-old woman, had a 30-year history of asthma. She underwent an anterior resection for carcinoma of the rectum in 1965 and a mastectomy for carcinoma of the breast in 1966. In April 1973,

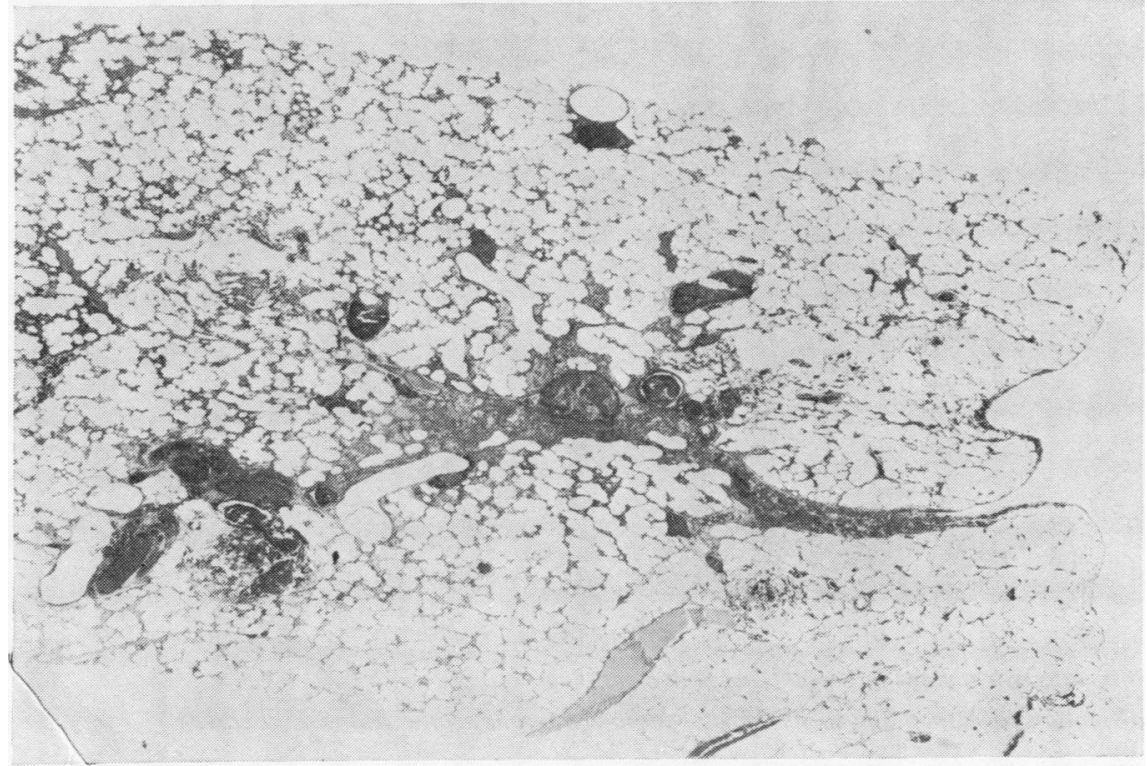

Fig 11 Case 6. Section from the posterior slice of the left lower lobe corresponding to the mid-zone band shadow showing atelectasis with pleural retraction. $H$ and $E \times 4.2$. 


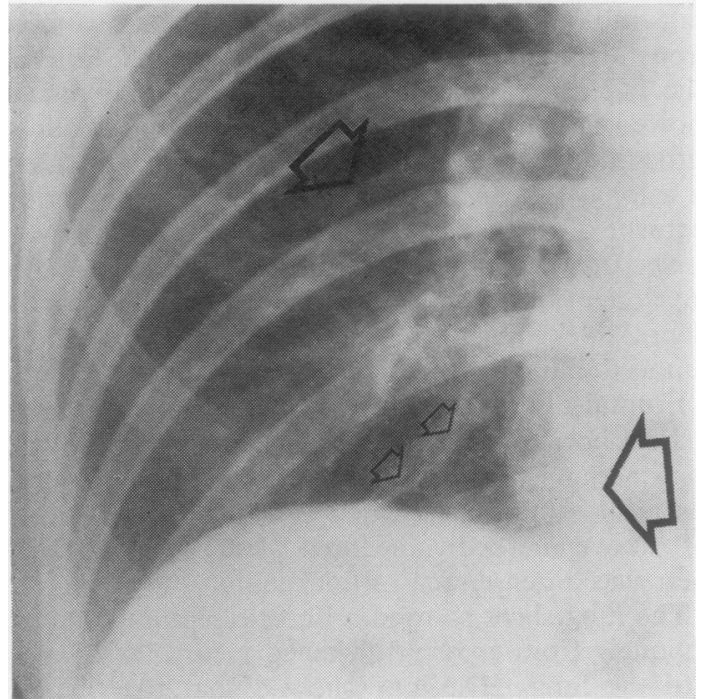

Fig 12 Case 7. In vivo chest radiograph shows mid-zone and basal band shadows. after a hysterectomy, she began to develop increas- $\frac{\stackrel{0}{*}}{\vec{*}}$

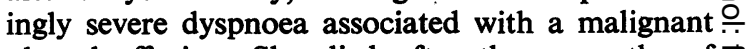
pleural effusion. She died after three months of $\overrightarrow{\vec{F}}$ progressive dyspnoea. Chest radiographs during her $\stackrel{5}{+}$ terminal illness showed a horizontal band shadow in the lingula associated with a left-sided pleural $\frac{\bar{\sigma}}{\overline{0}}$ effusion.

At necropsy no local recurrence of breast or rectal $\stackrel{\mathbb{\Omega}}{\circ}$ carcinoma was found but there was extensive peritoneal and subpleural tumour. The histology $\vec{\circ}$ showed a carcinoma consistent with a breast origin. The combined weight of the lungs was $835 \mathrm{~g}$.

Radiographs of the inflated lungs showed a band? shadow in the lingula, curving upwards towards the? pleura, associated with pleural retraction. At the ${ }_{0}$ periphery of the anterior aspect of the lingula there $v$ was a small area of consolidation. Small peripheral $\stackrel{\circ}{\circ}$ and subpleural metastases were present.

When the lungs were sliced there were scattered $\stackrel{\circ}{?}$ multiple subpleural metastases. In the lingula there $\vec{c}$ was a firm band-like lesion associated with deep indrawing between opposing pleural surfaces. Distal
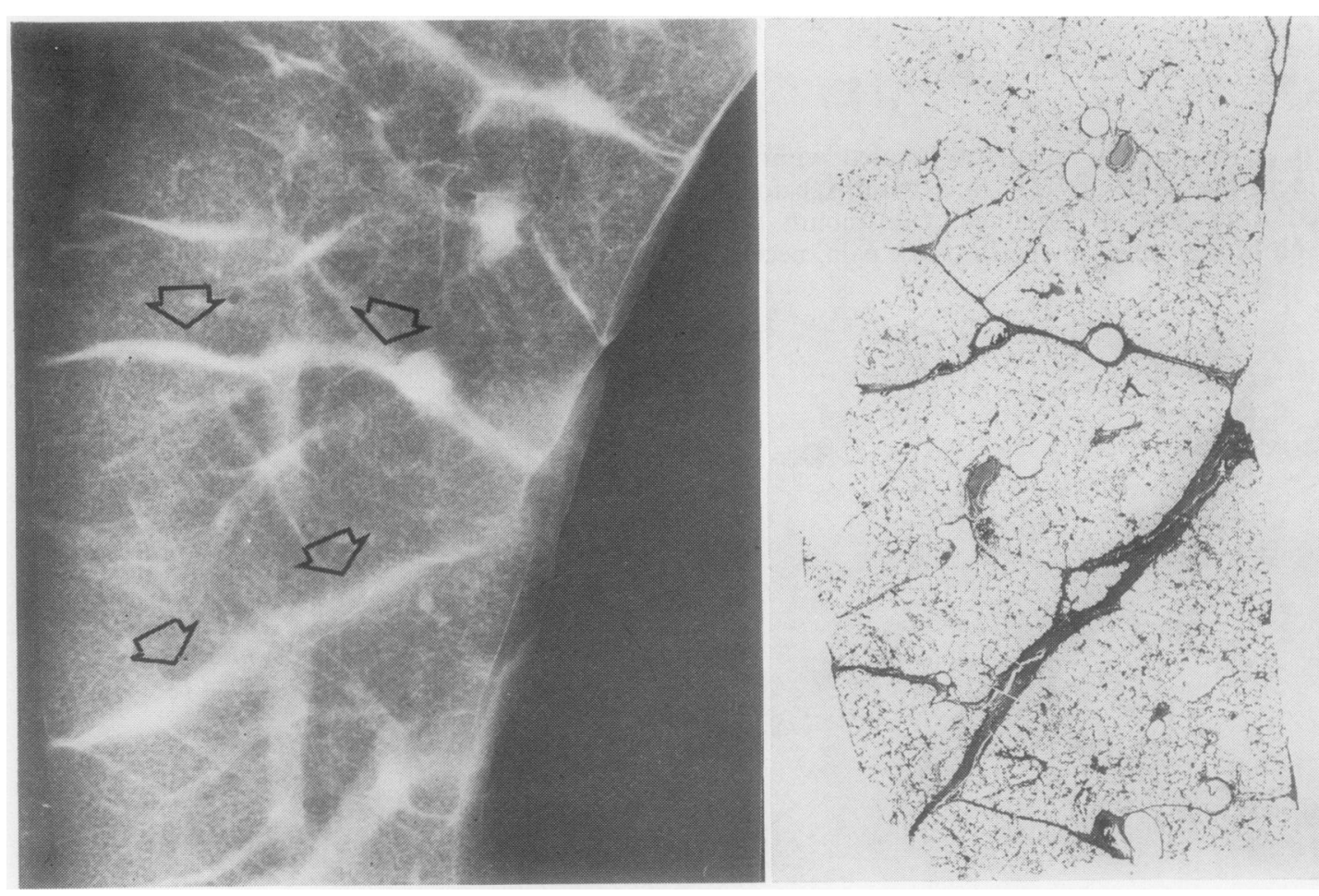

Fig 13

Fig 14

Fig 13 Case 7. Radiograph of lung slice showing broad band shadows.

Fig 14 Case 7. Histological section corresponding to fig 13 showing oedematous septa. $H$ and $E \times 1.8$. 


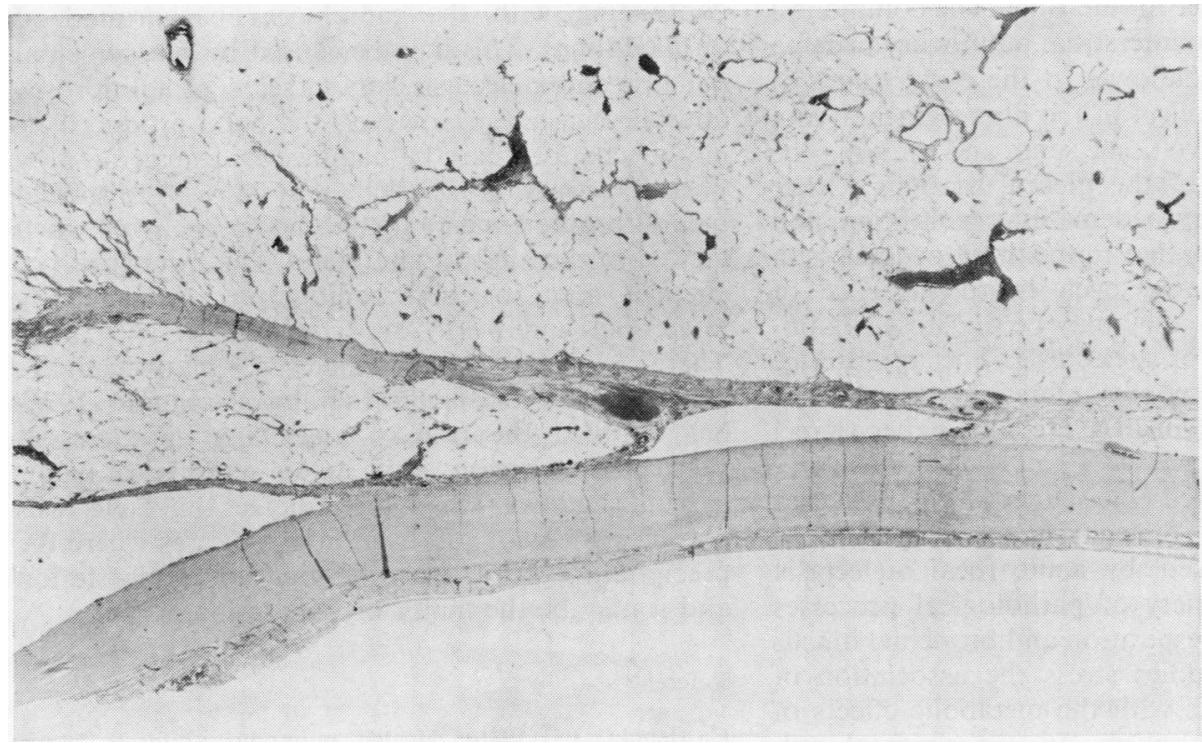

Fig 15 Case 8 . A fibrotic intralobar septum runs obliquely from the densely fibrotic diaphragmatic pleura. $\mathrm{H}$ and $E \times 3.7$.

to this the tip of the lingula was partially collapsed and consolidated.

Sections of the band-like lesion showed that it consisted of atelectatic lung between the pleural surfaces which were indrawn, fibrotic, and infiltrated with carcinoma. The collapsed parenchyma showed thickened alveolar walls and very marked alveolar wall and vascular elastosis. The tip of the lingula was partially collapsed. There was further pleural tumour, but in addition there were small fibrotic foci containing recanalized vessels, suggesting old infarction. In many of the sections there were fresh pulmonary emboli and also small bronchi plugged with mucus.

\section{Discussion}

\section{PERSISTENT BAND SHADOWS}

These studies demonstrate that infarct scars may produce persistent band shadows, as shown originally by Fleischner et al (1941). Infarct scars are contracted because the original lesion causes destruction of lung parenchyma and there is reparative fibrosis which may also produce indrawing of the pleural surface. They are thus more likely to produce linear or band shadows than the scarring of old pneumonia in which the alveolar architecture is maintained and organized intra-alveolar exudate leads to a solid scar without loss of tissue or distortion of the pleural profile.

In the cases described in this paper atelectasis plays an important role in the production of persist- ent band shadows either alone or in concert with other factors. It may be prominently associated with infarction (Fleischner et al, 1941; Fleischner, 1962). In case 1 atelectasis proximal to the infarct was responsible for the deep pleural indrawing noticed in the radiographs. Moreover, in cases 4 and 9 the main part of the band shadow itself was caused by atelectasis rather than the infarct scar itself. It is also possible for chronic atelectasis unassociated with infarction, scarring or pulmonary embolism to produce a persistent band shadow. In such cases the chronicity and irreversibility of the atelectasis may be indicated by thickening of the alveolar walls in the collapsed parenchyma (Spencer, 1968).

Intralobar septal fibrosis may also cause persistent band shadows resembling infarct scars on radiological examination. The origin of such fibrosis may be postinflammatory.

These observations stress that though pulmonary infarcts may cause longstanding linear shadows, atelectasis and intralobar fibrosis are also important causes, and that frequently more than one lesion is present.

TRANSIENT BAND SHADOWS

The genesis of transient shadows is more difficult to elucidate because their natural history is resolution. However, study of postmortem radiographs shows many band shadows present at death which are produced by potentially reversible lesions. By inference these could have been identified as transient shadows on in vivo films if radiographs had 
been taken at the appropriate time. The commonest finding was that acute interstitial pulmonary oedema may produce such shadows. In the early stages of oedema, fluid may collect in the intersegmental and interlobular connective tissue septa and there may be smooth indentation of the pleural margins. Other evidence of pulmonary oedema may be lacking, and unless it is realized that interstitial oedema can produce band shadows, even in the absence of classical Kerley lines, they may be diagnosed radiologically as infarcts or atelectasis. The appearance of line shadows caused by interstitial oedema has been described in detail in a previous paper (Kreel et al, 1975).

It has been suggested (Fleischner et al, 1941) that transient horizontal linear shadows above the diaphragm are caused by acute focal atelectasis resulting from a variety of pathological processes involving depressed respiration and bronchial mucus retention. Recent studies stress the association of acute focal atelectasis with the metabolic effects of acute pulmonary embolism. In experimental pulmonary embolism there is a shift of ventilation from unperfused to perfused lung (Robin, 1965). In his discussion of the underlying mechanisms he suggests that loss of surfactant in unperfused lung may be the basis of focal atelectasis in human pulmonary embolism: an increase in pulmonary surface tension, preceding atelectasis, occurs after experimental pulmonary artery occlusion (Finley et al, 1964). Pulmonary embolism causes bronchoconstriction (Gurewich et al, 1965) and alveolar duct constriction (Nadel, 1965) due in part to humoral factors and in part to local $\mathrm{CO}_{2}$ alterations (Severinghaus et al, 1961).

We did not find foci of atelectasis associated with acute pulmonary embolism in the absence of infarction but cannot exclude the possibility that such foci could be re-expanded by the positive pressure respiration which our technique involves, particularly if the atelectasis were caused by bronchoconstriction rather than bronchial obstruction. In the observed cases where acute atelectasis produced potentially reversible band shadows the cause was mechanical obstruction of airways due to purulent bronchiolitis.

The production of band shadows on chest radiographs depends on the visualization of linear areas contrasting with the radiolucent background of $\frac{\stackrel{0}{*}}{\overrightarrow{5}}$

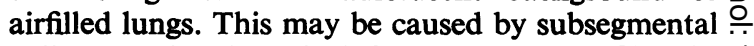
collapse and atelectasis, infarct scars, septal fibrosis, $\overrightarrow{\vec{F}}$ and oedema lines. However, the band produced by $\stackrel{\oplus}{\oplus}$ any mechanism may be manifest or exaggerated by pleural retraction so that the indrawn distorted $\frac{\bar{\sigma}}{\bar{\omega}}$ pleural profile appears within the parenchyma when $\overrightarrow{\widetilde{\sigma}}$ viewed in the appropriate plane. The mechanism of $\varrho$ changes seen in chest radiographs is frequently $\%$ inferential and based on sequential in vivo films. $\vec{O}$ There are relatively few direct correlative studies between pathologists and radiologists. Simon (1971) $\vec{\omega}$ has doubted the role of atelectasis and stressed $\frac{\mathscr{D}}{8}$ vascular obstruction as the major aetiological factor? in band shadows. This study indicates that atelectasis $\underset{0}{0}$ plays a significant role even in those cases where the $-v$ precipitating cause is a pulmonary vascular lesion $\stackrel{\circ}{\circ}$ and it may be the major or only lesion noted.

\section{References}

Castleman, B. (1940). Healed pulmonary infarcts. Arch. Path., 30, 130-142.

Finley, T. N., Tooley, W. H., Swenson, E. W., Gardner, R. E., and Clements, J. A. (1964). Pulmonary surface tension in experimental atelectasis. Amer. Rev. resp. Dis., 89, 372-378.

Fleischner, F. G. (1962). Pulmonary embolism. Clin. Radiol., 13, 169-182.

Fleischner, F. G., Hampton, A. O. and Castleman, B. (1941). ْి

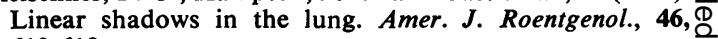
610-618.

Gurewich, V., Sasahara, A. A. and Stein, M. (1965). In $\overrightarrow{\vec{O}}$ Pulmonary Embolic Disease, edited by A. A. Sasahara and 3 M. Stein, pp. 162-169. Grune and Stratton, New York.

Kreel, L., Slavin, G., Herbert, A., and Sandin, B. (1975). Intralobar septal oedema: 'D' lines. Clin. Radiol., 26, 209-221.

Nadel, J. A. (1965). In Pulmonary Embolic Disease, edited? by A. A. Sasahara and M. Stein, pp. 153-161. Grune and $\frac{3}{3}$ Stratton, New York.

Robin, E. D. (1965). In Pulmonary Embolic Disease, edited by A. A. Sasahara and M. Stein, pp. 149-152. Grune and Stratton, New York.

Severinghaus, J. W., Swenson, E. W., Finley, T. N., Lategola, M. T., and Williams, J. (1961). Unilateral hypoventilation $D$ produced in dogs by occluding one pulmonary artery. J. appl. Physiol., 16, 53-60.

Simon, G. (1971). Principles of Chest X-ray Diagnosis, 3rdō edition. Butterworths, London.

Spencer, H. (1968). Pathology of the Lung, 2nd edition. Pergamon Press, London.

Wright, B. M., Slavin, G., Kreel, L., Callan, K., and Sandin, B. (1974). Postmortem inflation and fixation of humant lungs. Thorax, 39, 189-194. 